\title{
物联网技术在建筑智能化系统中的应用分析
}

\author{
钱洁君* \\ 厦门柏事特信息科技有限公司江苏分公司，江苏 210000
}

\begin{abstract}
摘 要: 不知不觉间物联网已经从一个模糊的概念变成了可以实现并加以使用的技术手段。基于物联网技术的发 展, 建筑行业中也出现了大量的智能化建筑。智能化建筑可以简化人们日常生活中一些繁琐的工作, 也能改善人们的 工作居住环境, 是未来建筑行业的主要发展方向。本文就当今物联网和建筑智能化系统的现状, 简述物联网技术在建 筑智能化系统的应用和存在的一些问题。
\end{abstract}

关键词：物联网；建筑；智能化；应用

\section{Application Analysis of Internet of Things Technology in Building Intelligent System}

\author{
Jie-Jun Qian* \\ Jiangsu Branch of Xiamen Best Information Technology Co., Ltd., Jiangsu 210000, China
}

\begin{abstract}
Unconsciously, the Internet of Things has changed from a vague concept to a technical means that can be realized and used. Based on the development of Internet of Things technology, a large number of intelligent buildings have emerged in the construction industry. Intelligent buildings can simplify some tedious work in people's daily life and improve people's working and living environment, which is the main development direction of the future construction industry. Based on the current situation of Internet of things and building intelligent system, this paper briefly introduces the application and existing problems of the Internet of Things technology in the building intelligent system and some existing problems.
\end{abstract}

Keywords: Internet of Things; architecture; intelligent; application

\section{一、前言}

现代化的智能系统是智能建筑中的重要组成部分, 智能建筑可以实现很多传统建筑所不能实现的功能。这些功能 通过物联网技术得以整合协调, 覆盖了建筑内的多个系统。系统与系统之间协同运行, 形成了一套自动化、一体化、 功能齐备的智能体系。物联网技术在建筑智能化系统中的应用充分体现了物联物技术的实践价值。

\section{二、物联网技术概述}

“智慧地球” 的概念驱动了物联网的诞生与发展，近年来受到越来越多的关注与认可 ${ }^{[1]}$ 。在物联网技术中，常见的 有射频识别 (RFID) 、扫描技术、传感器技术, 以互联网为核心, 具有完善并拓展建筑智能化的作用。作为建筑智 能化中的基础单元，物联网技术可以实现物品和物品的协同与互动，在确定建筑智能化的需求之后再启动物联网的运 行。物联网技术属于网络信息技术的一部分, 可根据智能建筑的实际需要将智能化功能整合并加以使用。在物联网的 作用下, 建筑智能化以物体为基准构成了一个物物相连的网络体系, 将智能建筑中的信息交流规则统一, 建立物品信 息。物联网技术的基础为传感器, 物品信息正是由相互传感去获取, 这些收集到的信息在物联网内进行处理, 使信息 识别和分析的效率大大提高, 优化了智能建筑的整体运营环境。

\section{三、建筑智能化系统}

建筑智能化系统是个庞大、繁复的体系，由大量分支系统构成，包含着多个运营子系统。现阶段，常见的子系统 有安防、消防、门禁等, 所有的子系统各有分工又相互协同, 共同维持着智能化建筑的运行。现代建筑工程发展趋势 已向建筑智能化逐渐倾斜。因目前还没有足够经验来制定一套统一标准, 所以在智能建筑的设计规划上, 应结合建筑 的实际需求, 来打造一个高效、优质的建筑环境, 不断提高建筑智能化系统的质量。建筑智能化简化了建筑运营的过 程, 将智能化功能进行整合, 为了让这种整合更具统一性和规范性, 在智能建筑中引入物联网技术是当今最有效的解 决方案 ${ }^{[2]}$ 。

*通讯作者: 钱洁君, 1990 年 07 月, 女, 汉, 江苏南京, 助理工程师, 学士。研究方向: 建筑智能化。 


\section{四、物联网技术在建筑智能化系统中的应用}

如图1所示，在现有的案例中，物联网技术在智能建筑中的应用主要有以下几个系统:

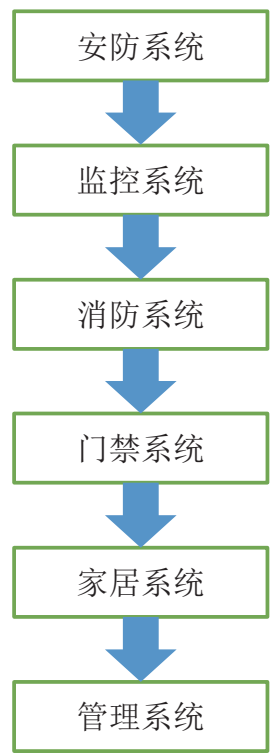

(一) 安防系统

图1 智能建筑中的系统

安防系统是建筑中最常见且最基础的部分。智能建筑中的安防系统也加人了物联网技术, 提高了建筑的安全 性, 为建筑内用户的安全提供了保障。安防系统的功能主要是人防、物防等。通过对智能建筑的监控, 将监控画 面传输到监控中心 (图2所示), 监控中心的工作人员可以通过监控画面了解到建筑的实时状况, 及时发现建筑中 存在的异常现象, 规避了可能发生的危险事故。在物联网技术的推动下, 要求智能建筑中的安防系统要具备快速发 现异常、快速发出警报的能力。例如：某智能小区中的安防系统在物联网技术的作用下安装了红外线探测器，用于 探测小区内的实时状况。物联网技术的应用将红外线探测器与其它系统相连, 如下文会提到的门禁系统和消防系统 等, 系统之间共同发挥作用。当红外线探测器发现一项系统出现危险时, 其他系统也会快速做出应对, 实现了系统 功能一体化 ${ }^{[3]}$ 。

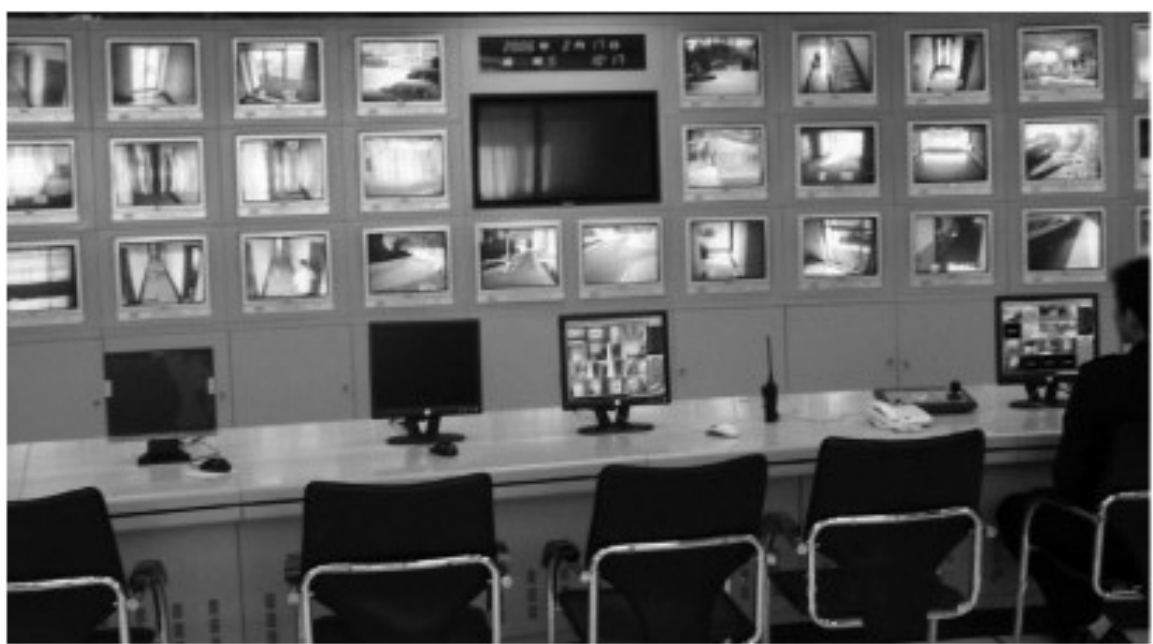

图2 监控中心

\section{(二) 监控系统}

顾名思义，监控系统的任务就是负责对智能建筑进行全方位监控，通过传输监控画面的方式保障小区的安全，即 利用物联网的技术将监控画面传到监控中心, 由监控中心判断建筑的安全状况。在监控系统中, 物联网技术可将所有 电气设备和多种分项系统相连接, 如通信系统、照明系统和电梯系统等。这些系统都处于监控系统的监测之下, 任何 
系统发生故障或运行不稳定都会及时反馈给监控系统, 有便于及时发现建筑内是否存在工作不正常的子系统。监控系 统会将异常信息报告给管理中心, 管理中心的维修人员会以最快速度赶到故障发生的地点, 及时修复, 维护智能化建 筑的正常运行 ${ }^{[4]}$ 。

\section{(三) 消防系统}

消防系统对于任何建筑来说都极其重要, 一套完整有效的消防系统可以避免火灾的发生和紧急处理突发的火灾事 故。智能建筑中的消防系统需要更好地担起预防火灾的职责并做出更为及时的处理。物联网技术在消防系统中的作用 非常明显, 它可以将整套消防系统中的传感器、报警装置、自动喷淋系统连接, 使消防系统科学、快速的对火灾进行 处理。建筑中火灾的发生十分常见, 大多是由于漏电或者煤气泄漏引起, 所以可在这些可能引起火灾的设备上安装一 个火灾探测器。和监控系统类似，物联网技术可将探测到的危险信号发回控制中心，并启动其它相应的消防子系统。 比如某一处发现异常并已检测到有明火存在, 即立即启动自动喷淋系统将火源及时熄灭 ${ }^{[5]}$ 。物联网技术下的智能建筑 在火灾的发现和预防上十分严谨, 发现火灾之后的处理也更效率、更规范, 为智能建筑在消防方面上的安全提供了极 大的保护。

(四) 门禁系统

一直以来, 建筑中的门禁系统都在研究, 如何做到在能发挥自身作用的同时变得更便利和全面一些, 物联网技术 很好地解决了这一问题。智能化建筑中的门禁系统会为业主配置具有REID射频识别功能的门禁卡, 这张门禁卡是业 主通过门禁系统的唯一凭据 ${ }^{[6]}$ 。门禁卡包含了业主如姓名、门牌号等这样的个人信息, 信息储存在物联网的数据库之 中。当业主出示门禁卡的时候, 这些信息会在数据库中检索, 只有业主信息和数据库信息能相互匹配的情况下, 门禁 系统才会对该业主开放。还有一种情况, 有些没有门禁卡的外来人员可以浑水摸鱼, 跟着上一位有门禁卡的业主偷偷 溜进小区。物联网技术考虑到了这种情况, 为保安人员配备了一种远程巡逻装置, 这种装置在50米之内的范围都能识 别到业主的门禁卡信息, 可以提早发现没有门禁卡的目标, 做好询问的准备。物联网技术让智能化建筑在门禁系统上 全面的保护着业主的出人安全，不仅如此还减轻了安保人员的工作量，大大提升了工作效率。

(五) 家居系统

智能家居系统是近几年新出现的高级智能系统，是人们通过物联网的发展逐渐摸索出来的产物，依赖于互联网的 数据传输和数据处理, 将家中的家电、安防系统、多媒体设备等连接到一起。作为智能化建筑中的衍生系统, 家居系 统从实际需要出发, 控制着建筑内的家居项目, 如暖通、照明、水电等, 可以对这些项目进行检测和控制。首先, 家 居系统会在用户的家中安装探测器和传感器用以对家居项目进行实时探测, 并把这些项目的状况以信息的方式发送给 一个接收终端（用户的手机或电脑）。用户在了解到信息之后可以利用物联网技术对这些项目进行控制, 例如发现家 中温度过高可开启空调; 湿度过低可开启加湿器; 还能检测空气中的煤气浓度, 预防了煤气泄漏的危险, 在预防火灾 上也起到了作用。理想中一整套的家居系统能帮助用户完成所有家居工作，用户只需要动一动手指就能完成对家中所 有家居项目的控制，如图3所示 ${ }^{[7]}$ 。

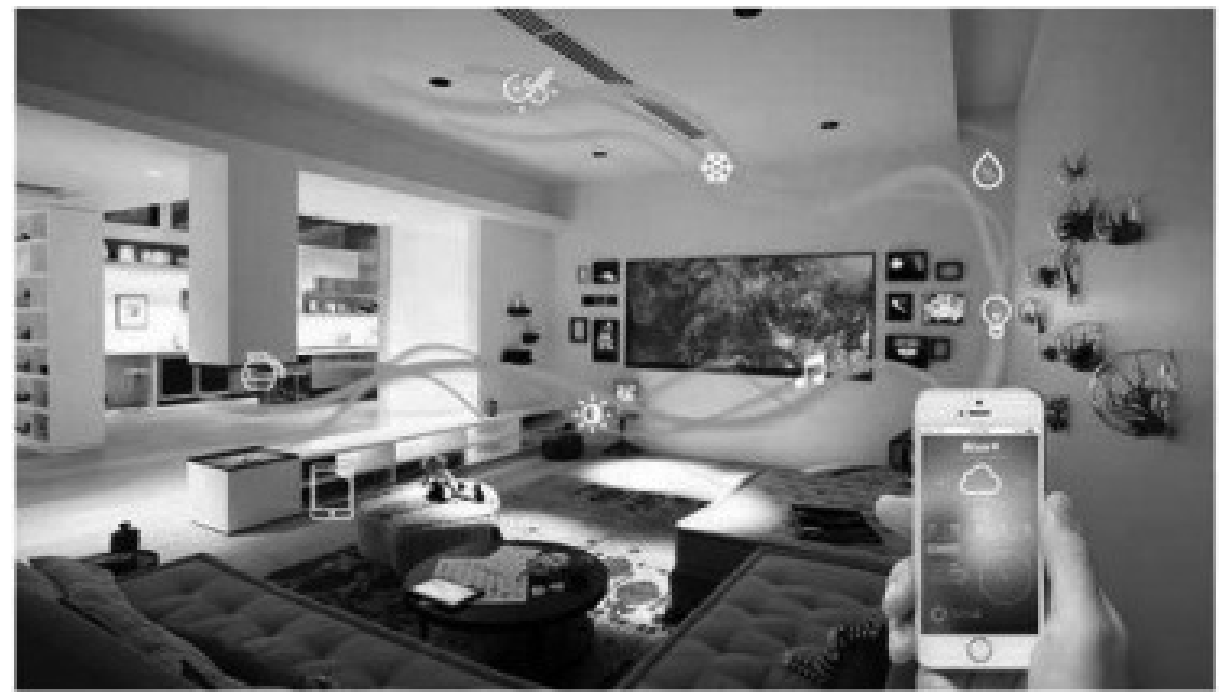

图3 理想中的智能家居 


\section{( 六) 管理系统}

管理系统可以对智能建筑中的公共设施进行管理，如停车位、电梯、给排水、配电设备等。物联网技术的RFID在 公共设施中安装了射频接收器, 给每个射频接收器编号, 由射频接收器收集公共设施中的信息, 再把信息传输到用户 及物业的控制中心。RFID主要利用图像识别技术对公共设施进行管理 ${ }^{[8]}$ 。例如我们熟知的车位信息显示，通常在小区 的车辆人口处, 配备了这样的装置, 准备进人小区的业主可以看到小区内还有多少空余车位。这就是物联网技术的作 用, 实现原理是通过给车位安装射频接收器来确认该车位是否有车辆停放。当电梯发生故障时, 物联网技术也能通过 射频接收器迅速发现, 确认发生故障的电梯之后及时切断该电梯的电力连接, 停止该电梯的运行, 避免了乘客在乘梯 过程中发生危险，随后上报给管理中心，维修人员将迅速对其进行修复。

\section{五、建筑智能化遇到的现实问题}

(一) 安全问题

1. 架构上的安全威胁: 作为智能化建筑的基础, 物联网技术的安全性决定了建筑能否正常顺利的运行, 然而物联 网技术在现如今还不够完善, 首先在构架上就存在一定的安全隐患。智能建筑中会使用大量的传感器, 通常来讲传感 器的感知节点设计相对简单, 计算能力有限, 协议和系统都不够安全, 使得传感器很容易遭到木马病毒的攻击。传感 器一旦遭到攻击, 很有可能向物联网中的其它关联系统发出错误的信息。系统与系统之间的关联性十分紧密, 一方出 错造成的连带关系可能造成整个智能系统的㿈疾。另外, 即便没有严重到系统㿈疾, 物联网中的信息遭窃取也会带来 极大的安全隐患。与互联网一样, 物联网之中也存储着海量的数据, 这些数据有一些设备的资料和用户的私人信息, 若被第三方获取并加以利用也会造成不小的损失。

2. 终端上的安全威胁: 终端上的安全问题主要体现在智能家居这一环节上。在智能家居之中终端是实现智能的常 用工。终端由用户操作, 由于用户的网络知识不同、操作水平不一, 造成了终端向物联网传递信息的不稳定, 可能会 对物联网的整体运行造成影响。此外，在终端上通常会验证用户的身份，传统的密钥形式的验证方式在互联网中就存 在着风险。另一种 “人脸” 或 “眼睛” 的认证方式在技术上无法保证每一次都能准确的识别用户身份，这给第三方提 供了可乘之机。

3. 通信上的安全威胁: 以手机为例, 作为智能系统的终端之一, 即便保证了设备的安全也难免会受到通信上的安 全威胁, 手机具有连接Web和收受信号的功能, 与智能系统连接的方式也是基于此。而这种信息数据通过无线传输的 模式, 在传输的过程中也容易遭到攻击而导致数据泄露。互联网中的主要工具是计算机, 计算机遭到攻击比较容易修 复。但在智能建筑中，物联网中包含建筑、设备、家居等等大量难以修复的元素，若被损毁后果可想而知。

综上所述，提高物联网的构架安全、加强通信协议安全是物联网技术发展中的重中之重。此外，智能家居与人的 生活息息相关，自然会涉及到许多法律问题。目前我国针对于物联网和智能化建筑的法律规定还不是十分完善, 需要 出台相应的法律法规, 对于物联网的网络犯罪严加控制和打击, 无论是用户和运营商都需要加强安全防护意识, 维护 智能建筑的安全运行 ${ }^{[9]}$ 。

\section{（二）建筑的成本控制}

物联网下的建筑智能化极大的提升了建筑的科学性, 实现了建筑自动化, 为建筑使用者带来了不同以往的全新体 验。但不容忽视的是, 其造价成本在现如今阶段非常之高, 任何一项智能系统的搭建都需要耗费大量资金。智能化建 筑的工程预算要合理安排，根据建筑目的来选取相应功能，不能盲目追求多功能，以防发生过去曾有过的投资商资金 链断裂现象 ${ }^{[10]}$

\section{六、结语}

物联网技术在建筑智能化系统中扮演着核心的角色，真正做到了“物物相连”，在实物之间建立了交流的通道。 在智能建筑中几乎在所有系统都用到了物联网技术, 使其运营功能更加完备, 为用户营造了一个智能、便利的生活工 作环境。但因其技术还在发展阶段, 许多问题逐渐浮出水面, 主要体现在工程预算和安全隐患上。相信随着技术手段 的不断完善和智能设备的普及，这些问题将被一一得到解决。就现阶段而言，物联网在建筑智能化系统的应用取得了 一定的成就，证明了物联网的实践价值，也表明这是未来智能建筑行业要坚持走下去的正确道路。

\section{参考文献:}

[1]刘英明.基于物联网技术的实训室智能管理系统设计[J/OL].电子技术与软件工程, 2019(20):44-45.

[2]王海鹏.物联网行业发展和业务应用研究[J].计算机产品与流通, 2019(10):60.

[3] 凌文青.物联网技术在建筑电气节能中的应用分析[J].湖北农机化, 2019(18):57.

[4]张羽.基于物联网的建筑结构监测方式分析 [J].福建建材, 2019(09):21-22. 
[5]方锐.物联网技术下智能建筑的成本控制与工程造价分析 [J]. 价值工程, 2019,38(26):58-59.

[6]郑立乾,孙风娇.关于物联网技术现状分析及应用前景探讨[J]. 计算机产品与流通, 2019(09):163.

[7]史国英.物联网技术在楼宇智能化系统中的应用 [J].智能建筑与智慧城市, 2018(10):58-59.

[8]杨秉陆.物联网技术在建筑智能化系统中的运用研究 [J].居舍, 2018(22):240-244.

[9]钮金杨小来.物联网技术在建筑智能化系统中的应用研究 [J]. 电子世界, 2017(21):56-58.

[10]裴涛.物联网技术在楼宇智能化系统中的应用 [J].智能建筑, 2017(01):14-15. 